\title{
Not Performed
}

National Cancer Institute

\section{Source}

National Cancer Institute. Not Performed. NCI Thesaurus. Code C141477.

An indicator that specifies that an activity did not occur. 